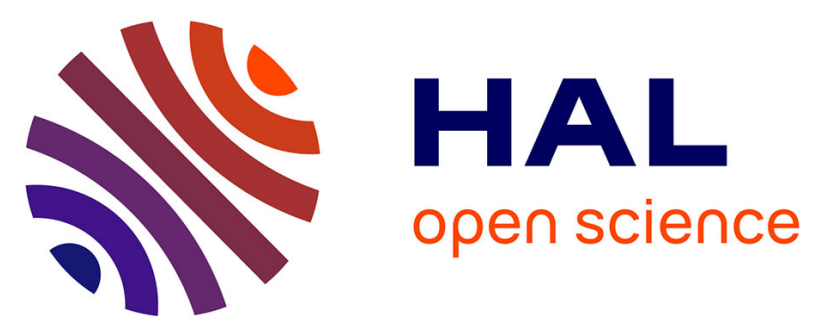

\title{
Performance analysis of four nonlinearity analysis methods using a model with variable complexity and application to uterine EMG signals.
}

Ahmad Diab, Mahmoud Hassan, Catherine Marque, Brynjar Karlsson

\section{To cite this version:}

Ahmad Diab, Mahmoud Hassan, Catherine Marque, Brynjar Karlsson. Performance analysis of four nonlinearity analysis methods using a model with variable complexity and application to uterine EMG signals.. Medical Engineering \& Physics, 2014, 36 (6), pp.761-7. 10.1016/j.medengphy.2014.01.009 . inserm-00995890

\section{HAL Id: inserm-00995890 https://www.hal.inserm.fr/inserm-00995890}

Submitted on 27 May 2014

HAL is a multi-disciplinary open access archive for the deposit and dissemination of scientific research documents, whether they are published or not. The documents may come from teaching and research institutions in France or abroad, or from public or private research centers.
L'archive ouverte pluridisciplinaire HAL, est destinée au dépôt et à la diffusion de documents scientifiques de niveau recherche, publiés ou non, émanant des établissements d'enseignement et de recherche français ou étrangers, des laboratoires publics ou privés. 
$\left\ulcorner\quad\right.$ Ahmad Diab $^{\mathrm{a}, \mathrm{b}, *}$, Mahmoud Hassan $^{\mathrm{c}}$, Catherine Marque ${ }^{\mathrm{a}}$ and Brynjar Karlsson ${ }^{\mathrm{b}}$

\section{Performance analysis of four nonlinearity analysis methods using a model with variable complexity and application to uterine EMG signals}

$\varepsilon$

âUR CNRS 7338, Biomécanique et Bio-ingénierie, Université de Technologie de Compiègne, Compiègne, France

${ }^{\mathrm{b}}$ School of Science and Engineering, Reykjavik University, Reykjavik, Iceland

'Laboratoire Traitement du Signal et de L’Image, INSERM, Université de Rennes1, Campus de Beaulieu, Rennes, France.

E-mail: ahmad.diab@utc.fr (A.Diab), mahmoud.hassan@univ-rennes1.fr (M.Hassan), catherine.marque@utc.fr (C. Marque), brynjar@ru.is (B. Karlsson).

\section{Abstract}

Several measures have been proposed to detect nonlinear characteristics in time series. Results on time series, multiple surrogates and their z-score are used to statistically test for the presence or absence of non-linearity. The z-score itself has sometimes been used as a measure of nonlinearity. The sensitivity of nonlinear methods to the nonlinearity level and their robustness to noise have rarely been evaluated in the past. While surrogates are important tools to rigorously detect nonlinearity, their usefulness for evaluating the level of nonlinearity is not clear. In this paper we investigate the performance of four methods arising from three families that are widely used in non-linearity detection: statistics (Time reversibility), predictability (Sample Entropy, Delay Vector Variance) and chaos theory (Lyapunov Exponents). We used sensitivity to increasing complexity and the Mean square Error (MSE) of Monte Carlo instances for quantitative comparison of their performances. These methods were applied to a Henon nonlinear synthetic model in which we can vary the complexity degree $(C D)$. This was done first by applying the methods directly to the signal and then using the z-score (surrogates) with and without added noise. The methods were then applied to real uterine EMG signals and used to 
distinguish between pregnancy and labor contraction bursts. The discrimination performances were compared to linear frequency based methods classically used for the same purpose such as Mean Power Frequency (MPF), Peak Frequency (PF) and Median Frequency (MF). The results show noticeable difference between different methods, with a clear superiority of some of the nonlinear methods (Time reversibility, Lyapunov exponents) over the linear methods. Applying the methods directly to the signals gave better results than using the z-score, except for Sample Entropy.

\section{Keywords}

Nonlinear time series analysis, uterine electromyogram, contraction discrimination, surrogates.

\section{Introduction}

One of the most common ways of obtaining information on neurophysiologic systems is to study the features of the signal(s) using time series analysis techniques. This traditionally rely on linear methods in both time and frequency domains [1]. Unfortunately, these methods cannot give information about purely nonlinear features of the signal. Due to the intrinsic nonlinearity of most biological systems, these nonlinear features may be present in physiological data and even be a characteristic of major interest. Recently, much attention has been paid to the use of nonlinear analysis techniques for the characterization of a biological signal [2]. Indeed, this type of analysis gives information about the nonlinear features of these signals, which arise from the underlying physiological processes, many of which have complex behavior. There is a growing literature reporting nonlinear analysis of various biosignal types (EEG [3], ECG [4], HRV [5] and EMG [6]).

The EHG or electrohysterogram (electrical uterine activity recorded on woman's abdomen) has been widely studied [7], [8], [9], [10], [11]. Nonlinear characteristics have been observed in the EHG and some success has been achieved by using these characteristics to obtain information of potential clinical usefulness. Radomski et al. show that nonlinear analysis of EHG based on the sample entropy statistic could differentiate dynamic states of uterine contractions [12]. A comparison between linear and nonlinear analysis with different conditions was done in [13]. It was concluded that median frequency is the best method among linear methods and that sample entropy is the best method among 
nonlinear methods for term/preterm EHG contractions classification. Sample entropy is superior to median frequency, which indicates that nonlinear analysis is more suitable than linear analysis for studying EHG signals. In [14] the progress of labor was evaluated using sample entropy. Our team has examined nonlinear EHG analysis methods. Our results confirm the presence of nonlinearity in EHG signals. This character of the signals is useful in discriminating between pregnancy and labor contractions [15], [2], [16]. Practical disadvantages of the nonlinear analysis methods have been reported in [16]. They include excessive calculation time due to surrogates analysis and promising but inconclusive results due to the small amount of data that can practically be used due to heavy calculation times.

This paper presents work that extends previous work done in our group in comparing Approximate Entropy, Correntropy and Time reversibility [16]. In this work we implemented additional nonlinear analysis methods (delay vector variance, Lyapunov exponents) and new ways of testing them. We also used a larger database of real signals than in the previous work and we investigated the sensitivity of the methods to the varying complexity of signals and their robustness. The kind of sensitivity and robustness analyses of non-linearity measures presented in this paper, are rare or absent in the literature.

Four nonlinear methods: Time reversibility [17], Sample Entropy [18], Delay Vector Variance [19] and Lyapunov Exponents [20] were used in this work. Sensitivity of these methods to the complexity degree $(C D)$ of a signal as well as robustness analysis were done on Henon model synthetic signals where $C D$ can be controlled. The sensitivity to $C D$ was first studied using the direct value provided by the method. It was then studied using surrogates and z-score, as the measure permitting evaluation of the nonlinearity. One objective of this study is to show which method(s) is most sensitive to the change of signal complexity. A second objective is to determine whether the use of surrogates gives better overall results than the direct application of the methods. This is of major practical importance for clinical application, as the generation of surrogates is very computationally expensive. The methods are also compared using the Mean square error (MSE) of the method results for 30 Monte Carlo instances of the signal. Finally, these non-linear methods are compared to three linear frequency 
based characteristics of the signal, MPF, PF and MF, when applied to real EHG signals, in order to discriminate pregnancy and labor contractions.

\section{Materials and Methods}

\section{A) Data}

\section{Synthetic signals}

The Henon map is a well-known two-dimensional discrete-time system given by:

$$
\begin{gathered}
Y_{t+1}=c-Y_{t}^{2}+C D * X_{t}, \\
X_{t+1}=Y_{t},
\end{gathered}
$$

where $Y_{t}$ and $X_{t}$ represent dynamical variables, $C D$ is the complexity degree and $c$ is the dissipation parameter. In this paper we use $c=1$ as in [21] and $C D \in[0,1]$ to change the model complexity [22] (Figure 1). The number of generated points is fixed to 1000 . For the robustness analysis, we add to the synthetic signals a white Gaussian noise with the same duration, with a fixed 5db SNR with $C D$ varying between 0 and 1 with a step 0.1 . In the Monte Carlo analysis, we use 30 signals generated for each $C D$ value.

\section{Real signals}

EHG signals were recorded from 38 subjects using a $4 \times 4$ electrode matrix located on the subject's abdomen (Figure 2), during one hour either at rest (woman lying on a bed) or during labor. One signal channel (bipolar vertical 7: BP7), located on the median vertical axis of the uterus was used for subsequent analysis (see [23] for details). After segmentation we obtained 115 labor bursts (recorded during delivery) and 174 pregnancy bursts (recorded more than 24 hours before delivery).

\section{B) Non-linear Analysis Methods}

\section{Statistics family}


99 A time series is said to be reversible only if its probabilistic properties are invariant with respect to 1.. time reversal. Time irreversibility can be taken as a strong signature of nonlinearity [17]. In this paper 1.1 we used the simplest method, described in [24] to compute the time reversibility of a signal $S_{n}$ :

$$
\operatorname{Tr}(\tau)=\left(\frac{1}{N-\tau}\right) \sum_{n=\tau+1}^{N}\left(S_{n}-S_{n-\tau}\right)^{3}
$$

1.r where $N$ is the signal length and $\tau$ is the time delay.

\section{1.r 2. Chaos theory family}

\section{$1 \cdot \varepsilon \quad$ a) Lyapunov Exponents}

1.0 Lyapunov exponent (LE) is a quantitative indicator of system dynamics, which characterizes the 1.7 average convergence or divergence rate between adjacent tracks in phase space [20]. We used the 1.V method described in [13] to compute LE:

$$
\lambda=\lim _{t \rightarrow \infty} \lim _{y_{y_{0}} \| \rightarrow 0}\left(\frac{1}{t}\right) \log \left(\left\|\Delta_{y_{t}}\right\| /\left\|\Delta_{y_{0}}\right\|\right)
$$

1.1 Where $\left\|\Delta_{y_{0}}\right\|$ and $\left\|\Delta_{y_{t}}\right\|$ represent the Euclidean distance between two states of the system, 1.9 respectively to an arbitrary time $t_{0}$ and a later time $t$.

\section{3. Predictability family}

111 a) Sample Entropy

II Sample Entropy (SampEn) is the negative natural logarithm of the conditional probability that a

114 dataset of length $N$, having repeated itself for $m$ samples within a tolerance $r$, will also repeat itself for II $m+1$ samples. Thus, a lower value of SampEn indicates more regularity in the time series [18]. We 110 used the method described in [12] to compute SampEn: 
117 For a time series of $N$ points, $x_{1}, x_{2}, \ldots, x_{N}$, we define subsequences, also called template vectors, of

IIV length $m$, given by: $y_{i}(m)=\left(x_{i}, x_{i+1}, \ldots, x_{i+m-1}\right)$ where $i=1,2, \ldots, N-m+1$.

$11 \wedge$ Then the following quantity is defined: $B_{i}^{m}(r)$ as $(N-m-1)^{-1}$ times the number of vectors $X_{j}^{m}$ within $r$ 119 of $X_{i}^{m}$, where $j$ ranges from 1 to $N-m$, and $j \neq i$, to exclude self-matches, and then define:

$$
B^{m}(r)=\frac{1}{N-m} \sum_{i=1}^{N-m} B_{i}^{m}(r)
$$

1r. Similarly, we define $A_{i}^{m}(r)$ as $(N-m-1)^{-1}$ times the number of vectors $X_{j}^{m+1}$ within $r$ of $X_{i}^{m+1}$, where $j$ ranges from 1 to $N$ - $m$, where $j \neq i$, and set

$$
A^{m}(r)=\frac{1}{N-m} \sum_{i=1}^{N-m} A_{i}^{m}(r)
$$

I r The parameter $\operatorname{SampEn}(m, r)$ is then defined as $\lim _{N \rightarrow \infty}\left\{-\ln \left[A^{m}(r) / B^{m}(r)\right]\right\}$, which can be estimated irr by the statistic:

$$
\operatorname{SampEn}(m, r, N)=-\ln \left[A^{m}(r) / B^{m}(r)\right]
$$

I $Y \varepsilon \quad$ where $N$ is the length of the time series, $m$ is the length of sequences to be compared, and $r$ is the tolerance for accepting matches.

\section{b) Delay Vector Variance}

I rv We use the measure of unpredictability $\sigma^{* 2}$ described in [25]:

I $\mathrm{k}$ Time series can be represented in phase space using time delay embedding. When time delay is I 9 embedded into a time series, it can be represented by a set of delay vectors (DVs) of a given 1ץ. dimension $m$. The dimension of the delay vectors can then be expressed as $X(k)=\left[x_{(k-m \tau)} \ldots x_{(k-\tau)}\right]$, IT) where $\tau$ is the time lag. For every DV $X(k)$, there is a corresponding target, namely the next sample $x_{k}$. Irr A set $\beta_{k}(m, d)$ is generated by grouping those DVs that are within a certain Euclidean distance $d$ to Tr $X(k)$.This Euclidean distance will be varied in a standardized manner with respect to the 
I 1 \& distribution of pairwise distances between DVs. For a given embedding dimension $m$, a measure of iro unpredictability $\sigma^{* 2}$ (target variance) is computed over all sets of $\beta_{k}$.

Irฯ The mean $\mu_{d}$ and the standard deviation $\sigma_{d}$ are computed over all pair wise Euclidean distances IrV between DVs given by $\|x(i)-x(j)\|(i \neq j)$. The sets $\beta_{k}(m, d)$ are generated such that $\beta_{k}=$ Ir^ $\{x(i) \backslash\|x(k)-x(j)\| \leq d\}$ i.e., sets which consist of all DVs that lie closer to $X(k)$ than a certain i 9 distance $d$, taken from the interval $\left[\mu_{d^{-}} n_{d *} \sigma_{d} ; \mu_{d}+n_{d^{*}} \sigma_{d}\right]$ where $n_{d}$ is a parameter controlling the span I๕. over which to perform DVV analysis.

Iミ) For every set $\beta_{k}(m, d)$ the variance of the corresponding targets $\sigma_{k}^{2}(m, d)$ is computed. The average I $\left\{r \quad\right.$ over the $N$ sets $\beta_{k}(m, d)$ is divided by the variance of the time series signal $\sigma_{x}^{2}, \sigma_{k}$ gives the inverse I $\leqslant$ measure of predictability, namely target variance $\sigma^{*^{2}}$.

$$
\sigma^{* 2}=\frac{(1 / N) \sum_{k=1}^{N} \sigma_{k}^{2}}{\sigma_{x}^{2}}
$$

\section{$l \leq \varepsilon \quad$ C) Surrogates and z-score.}

Iso The most commonly used null hypothesis considers that a given time series is generated by a Gaussian I $\leqslant 7$ linear stochastic process and collected through a nonlinear measurement static function. Thus $I \leqslant \vee$ surrogates must have the same linear properties (autocorrelation and amplitude distribution) as the I $\wedge$ original signal. However, any underlying nonlinear dynamic structure within the original data is $1 \leqslant 9$ altered in the surrogates by phase randomization [16].

10. The statistics of significance $\mathrm{z}$-score is,

$$
Z_{\text {score }}=\frac{\left|q_{0}-\left\langle q_{s}(i)\right\rangle\right|}{\sigma_{q}(i)}
$$
where $q_{0}$ stands for the statistic on the original time series, $\left\langle q_{s}(i)\right\rangle$ for the mean and $\sigma_{q}(i)$ for the standard deviation of the surrogate, for $i=1,2, \ldots, M$ (number of generated surrogate). The critical value of z-score is 1.96 [26]. 


\section{A) Results on synthetic signals}

In this section we study the evolution of the values generated by the four methods with variable complexity degree $(C D)$ of the Henon synthetic model in four cases; 1) direct application of the method with no added noise, 2) using surrogates with no added noise, 3) direct application of the method with added noise and 4) using surrogates with added noise. The added noise is a white Gaussian noise $(\mathrm{SNR}=5 \mathrm{db}$ ) while $C D$ varies between 0 and 1 , for the Henon model. Our first objective is to test the sensitivity of the methods to varying $C D$ for signals with and without noise. The use of surrogates is computationally very expensive and therefore our second objective is to test if the use of surrogates improves the method sensitivity or not. .

We compare the methods using two criteria, the method's sensitivity to the change of $C D$ (slope of the curve "value of the method" vs. "CD") and the MSE of the method for different values of $C D$.

Figure 3-A1 presents the mean value for each method (direct method value) as a function of $C D$ computed from the 30 Monte Carlo instances of the signal generated by the Henon model. Figure 3A2 presents the MSE of the methods for each $C D$. We see in Figure 3-A1 that in the direct case without noise, the four methods evolve well but with differences in their sensitivity (slopes). Tr and LE are more sensitive than the other methods. In Figure 3-A2 we observe that Tr has a much lower MSE than LE.

Figure 3-B1 presents the effect of adding noise $(\mathrm{SNR}=5 \mathrm{db})$ on the methods. We notice no significant slope for the LE and SampEn. The sensitivity of Tr and DVV also decreases with the addition of noise. In the other hand we find, Figure 3-B2, that DVV and SampEn give the lowest MSE. However SampEn does not demonstrate any sensitivity to the variation of $C D$ so this method is useless for the noisy signal. Tr gives an intermediate MSE and the highest sensitivity when compared to the other methods when applied to noisy signals. 
11. We then applied the methods to the synthetic signals with surrogates using the z-score as measure, in order to test if the use of surrogates improves the results or not. Figure 3-C1 presents the $\mathrm{z}$-score for each method versus $C D$. We note that all the methods reflect the non-linearity of the signal generated by the Henon model as theirs z-score are always above 1.96 . In terms of sensitivity to $C D$ variation, SampEn is the best, but with the highest MSE (Figure 3-C2). Tr presents an acceptable evolution for lower $C D$. But beyond $C D=0.4$ an unexpected decrease occurs in the curve and the $\operatorname{Tr}$ value remains constant after $C D=0.7$. This method however, gives the lowest MSE (Figure 3-C2). The DVV method presents an intermediate slope, contrary to the LE that presents no change with $C D$. Both DVV and LE have low MSE under these conditions.

The methods were then applied to the signals using again surrogates and z-score but with added noise ( $\mathrm{SNR}=5 \mathrm{db}$ ). All the methods still reveal the nonlinearity of the model. Indeed z-score is above 1.96 for all the methods, except for DVV where it gives a z-score value lower then 1.96 for $C D$ between 0.4 and 0.6. We can clearly notice an increase in the sensitivity of Tr, Figure 3-D1, compared to the case in Figure 3-C1. SampEn has a good evolution beyond $C D=0.4$ but, on the other hand, it presents a rapid increase in MSE (Figure 3-D2). The LE and DVV do not evolve as a function of $C D$ (Figure 3D1) and give similar MSE as $\operatorname{Tr}$ (Figure 3-D2).

\section{B) Results on real signals}

The different nonlinear methods were applied to real uterine EMG signals (EHG), first direct application of the method, and then with surrogates. We also computed three classical linear frequency based parameters from these real signals. The values were then used to discriminate the pregnancy and labor contractions. We used ROC curves in order to test the discriminating power of each case.

Our first objective was to test if the use of surrogates improves the discrimination of EHG bursts recorded during Pregnancy or Labor. Our second objective was to compare the performances of linear and nonlinear methods and to verify that the nonlinear methods reveal the evolution of EHG characteristics better than the linear ones. The ROC curves obtained with the different methods without and with use of surrogates are presented Figure 4-B and Figure 4-C respectively. The 
$r \cdot \uparrow$ characteristics of all the ROC curves without and with use of surrogates are presented in Table I and

$r \cdot \vee \quad$ Table II respectively. From these data, it is clear that nonlinear methods improve the discrimination of $r \cdot \wedge$ pregnancy and labor signals. Indeed, the highest Area Under Curve AUC (0.842), sensitivity (0.86) r.9 and specificity (0.72) are obtained for the Tr method whatever the nonlinear or linear methods used. r. The MPF and LE methods also give an acceptable performance (Figure 4-B) with AUC $=0.778$ and r) $\mathrm{AUC}=0.758$ respectively. The performances in correct discrimination of labor varies markedly from rir AUC $=0.478$ with SampEn to AUC $=0.842$ with $\mathrm{Tr}$. When surrogates are used, all ROC curves present YIT approximately the same appearance with the highest AUC $=0.650$ obtained for SampEn. Using Y) surrogates we notice that the performance of SampEn improves while that of DVV remains Y10 approximately the same. On the other hand, the performance of $\operatorname{Tr}$ and LE seem to decrease with the YIT use of surrogates. Finally, we can conclude from Figure 4 and Table I that nonlinear methods can YIV provide better discrimination between pregnancy and labor contractions compared to the linear Y1 methods. Furthermore, even if the use of surrogates improves the performance of some methods, it r19 does not generally improve the discrimination results.

\section{rr. IV. Discussion and conclusion}

rr) We analyzed, quantitatively and as comprehensively as possible, four different nonlinear analysis rrr methods (Tr, SampEn, DVV and LE). These methods were applied on synthetic signals, in order to rrr test their sensitivity to the change in signal complexity, in normal and noisy conditions, with or rrs without using surrogates. All four methods were found to reflect correctly the increasing complexity rro of the signals in the noise free case, but with different sensitivities. In the case of added noise and rצ direct application of the method, as expected, a decrease in the sensitivity of all methods occurred at a rrv low Signal to Noise Ratio $(\mathrm{SNR}=5 \mathrm{db})$. Indeed, at this low SNR, none of the methods detected the r^A varying complexity of the signal, except for $\mathrm{Tr}$, which clearly reflected the increasing non-linearity. rrq The sensitivity of SampEn increased with the use of surrogates and it gave the highest sensitivity of all $r$ r. the methods, in the case of surrogate use with no added noise. Indeed SampEn has previously been shown to be sensitive to many aspects of the signal characteristics, including the sampling rate of the signal [14], [11]. Unexpected results were obtained in the case of surrogate use and with added noise. 
$\operatorname{Tr}$ was more sensitive when compared to the previous case, and SampEn still presented a good sensitivity. We noticed that in the case of surrogate use, SampEn gave the highest sensitivity but also had the highest MSE, making it unreliable.

In this paper we also presented results obtained using nonlinear and linear methods for discrimination of EHG bursts recorded during pregnancy and labor. Comparison between the methods indicated that $\mathrm{Tr}$, which is a nonlinear method, applied without using surrogates is clearly better in discriminating correctly pregnancy and labor contractions than the other methods. We can see also that the use of surrogates improves the performance of some methods like SampEn. These results confirm the results obtained during the study on synthetic signal, since the sensitivity of SampEn increases if surrogates are used, a posteriori justifying the use of the Henon model.

To sum up, the main findings of this study are the following: (i) Some of the studied methods are insensitive to varying signal complexity; (ii) SampEn performance depends on the use of surrogates; (iii) Generally speaking, none of the studied methods performed best in all the studied situations; (iv) Tr is very sensitive to change of model complexity, giving average or good performances, associated with the lowest MSE in most situations.

This leads to the conclusion that, of the four methods tested, Tr performed best for our application on real EHG. Indeed Tr deals robustly with real, usually noisy, signals and has a good sensitivity to complexity, one of the EHG characteristics that permits discrimination of uterine contraction efficiency. Using surrogates and the z-score, as a measure of nonlinearity, does not seem to bring any improvement to Tr. Therefore we will not use them for further work on EHG when using Tr.

There are some weaknesses in our study of which we are aware and aim to improve. $\mathrm{Tr}$ is dependent on the length of the signal and on the choice of the time delay $(\tau)$ and we aim to find a method to optimize these parameters. In further work we also aim to use all of the available bipolar channels $(\mathrm{VA} 1, \ldots, \mathrm{VA} 12)$ instead of only one channel, as in this work. This has been shown to dramatically increase the discrimination rate as evidenced in prior work [27].

\section{Acknowledgment:}


ro9 The authors wish to thank Dr. Jeremy Terrien for his helpful advice.

rฯ. Conflict of interest

iт) No conflict of interest.

rqr Sources of funding for research

French Ministry of Research, French ministry of foreign affair and the EraSysBio+ program.

rฯะ Ethical Approval

rro The measurements in Iceland were approved by the relevant ethical committee (VSN 02-0006-V2),

Yฯ7 those in France approved by the regional ethical committee (ID-RCB 2011-A00500-41) of Amiens riv Hospital.

rฯ人

rาq

$r Y$.

rVI

rVY

rVT

rVะ

rVo

rVT

YVY

rVA 


\section{References:}

ץ^. [1] Bendat, J.S. and Piersol, A.G., Random Data Analysis and Measurement Procedures. Measurement Science and Technology, 2000. 11(12): p. 1825.

[2] Hassan, M., Terrien, J., Alexandersson, A., Marque, C., and Karlsson, B., Nonlinearity of EHG signals used to distinguish active labor from normal pregnancy contractions. in Conf Proc IEEE Eng Med Biol Soc. 2010, p. 2387-2390.

[3] Takahashi, T., Cho, Raymond Y., Mizuno, T., Kikuchi, M., Murata, T., Takahashi, K., and Wada, Y., Antipsychotics reverse abnormal EEG complexity in drug-naive schizophrenia: A multiscale entropy analysis. NeuroImage, 2010. 51(1): p. 173-182.

[4] Shiogai, Y., Stefanovska, A., and McClintock, P.V.E, Nonlinear dynamics of cardiovascular ageing. Physics Reports, 2010. 488(2-3): p. 51-110.

[5] Mohebbi, M. and Ghassemian, H., Prediction of paroxysmal atrial fibrillation based on nonlinear analysis and spectrum and bispectrum features of the heart rate variability signal. Computer Methods and Programs in Biomedicine, 2012. 105(1): p. 40-49.

[6] Xie, H.-B., Zheng, Y.-P., Guo, J.-Y., and Chen, X., Cross-fuzzy entropy: A new method to test pattern synchrony of bivariate time series. Information Sciences, 2010. 180(9): p. 1715-1724.

[7] Lucovnik, M., Kuon, R. J., Chambliss, L. R., Maner, W. L., Shi, S. Q., Shi, L., Balducci, J., Garfield, R. E., Use of uterine electromyography to diagnose term and preterm labor. Acta Obstet Gynecol Scand, 2011. 90(2): p. 150-7.

[8] Garfield, R.E., Maner, W. L., MacKay, L. B., Schlembach, Dietmar, Saade, G. R., Comparing uterine electromyography activity of antepartum patients versus term labor patients. American Journal of Obstetrics and Gynecology, 2005. 193(1): p. 23-29.

[9] Schlembach, D., Maner, W. L., Garfield, R.E., Maul, H., Monitoring the progress of pregnancy and labor using electromyography. Eur J Obstet Gynecol Reprod Biol, 2009. 144 Suppl 1: p. S33-9.

[10] Hassan, M., Alexandersson, A., Terrien, J., Muszynski, C., Marque, C., Karlsson, B., Better pregnancy monitoring using nonlinear propagation analysis of external uterine 
electromyography. IEEE Transactions on Biomedical Engineering, April 2013, Vol 60(4): p. $1160-1166$.

[11] Diab, A., Hassan, M., Karlsson, B., Marque, C., Effect of decimation on the classification rate of nonlinear analysis methods applied to uterine EMG signals. IRBM journal, 2013 (In press).

[12] Radomski, D., Grzanka, A., Graczyk, S., and Przelaskowski, A., Assessment of Uterine Contractile Activity during a Pregnancy Based on a Nonlinear Analysis of the Uterine Electromyographic Signal. Information Technologies in Biomedicine, 2008: p. 325-331.

[13] Fele-Zorz, G., Kavsek, G., Novak-Antolic, Z., and Jager, F., A comparison of various linear and non-linear signal processing techniques to separate uterine EMG records of term and pre-term delivery groups. Med Biol Eng Comput, 2008. 46(9): p. 911-22.

[14] Vrhovec, J., Evaluating the progress of the labour with sample entropy calculated from the uterine EMG activity. Elektrotehniski vestnik-Electrotechnical Review, 2009. 76(4): p. 165-170.

[15] Diab, A., Hassan, M., Marque, C., and Karlsson, B., Quantitative performance analysis of four methods of evaluating signal nonlinearity: Application on uterine EMG signals, in Conf Proc IEEE Eng Med Biol Soc. 2012.

[16] Hassan, M., Terrien, J., Karlsson, B., and Marque, C., Comparison between approximate entropy, correntropy and time reversibility: Application to uterine EMG signals, Medical Engineering \& Physics (MEP), oct 2011, vol. 33, p. 980-986.

[17] Diks, C., van Houwelingen, J. C., Takens, F., and DeGoede, J., Reversibility as a criterion for discriminating time series. Physics Letters A, 1995. 201(2-3): p. 221-228.

[18] Richman, J.S. and Moorman, J.R., Physiological time-series analysis using approximate entropy and sample entropy. American Journal of Physiology-Heart and Circulatory Physiology, 2000. 278(6): p. H2039-H2049.

[19] Gautama, T., Mandic, D.P., and Van Hulle, M.M., The delay vector variance method for detecting determinism and nonlinearity in time series. Physica D: Nonlinear Phenomena, 2004. 190(3-4): p. 167-176. 
[20] Wolf, A., Swift, Jack B., Swinney, Harry L., Vastano, and John A., Determining Lyapunov exponents from a time series. Physica D: Nonlinear Phenomena, 1985. 16(3): p. 285-317.

[21] Pincus, S.M., Approximate Entropy as a Measure of System-Complexity. Proceedings of the National Academy of Sciences of the United States of America, 1991. 88(6): p. 2297-2301.

[22] Casas, G.A. and Rech, P.C., Multistability annihilation in the Hénon map through parameters modulation. Communications in Nonlinear Science and Numerical Simulation, 2012. 17(6): p. $2570-2578$.

[23] Karlsson, B., Terrien, J., Guðmundsson, V., Steingrímsdóttir, T., and Marque, C., Abdominal EHG on a 4 by 4 grid: mapping and presenting the propagation of uterine contractions. in 11th Mediterranean Conference on Medical and Biological Engineering and Computing, 2007, p. 139425, Ljubljana, Slovenia.

[24] Schreiber, T. and Schmitz, A., Surrogate time series. Phys. D, 2000. 142(3-4): p. 346-382.

[25] Kuntamalla S., R.L., The Effect of Aging on Nonlinearity and Stochastic Nature of Heart Rate Variability Signal Computed using Delay Vector Variance Method. International Journal of Computer Applications, 2011. Volume 14(No.5): p. 40-44.

[26] Slađana, S., Surrogate data test for nonlinearity of the rat cerebellar electrocorticogram in the model of brain injury. Signal Processing, 2010. 90(12): p. 3015-3025.

[27] Hassan, M., Terrien, J., Alexandersson, A., Marque, C., and Karlsson, B., Improving the classification rate of labor vs. normal pregnancy contractions by using EHG multichannel recordings. in Conf Proc IEEE Eng Med Biol Soc. 2010, p. 4642-4645. 
ry.

(4)

rur

זד

Mบ

r.

rาy

ry

MTN

rาq

rV.

rv

TVY
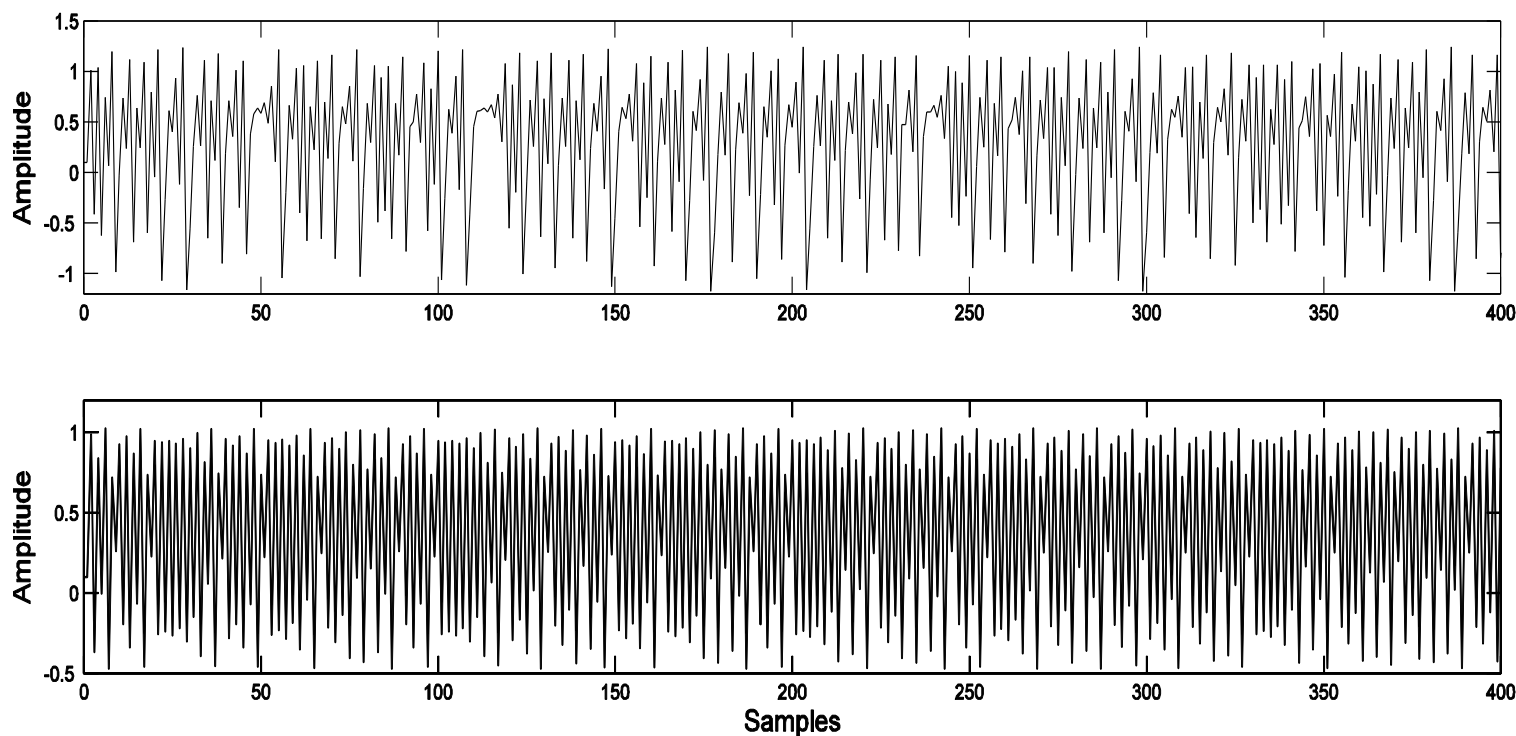

Fig. 1. Simulated signal generated using Henon model with different complexity degrees $(C D)$. Top: $C D=0.1$, Bottom: $C D=0.9$ 


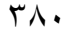

HN

rNT

rᄉr

T人ร

rNo

ए人т

TNV

rᄉ⿵
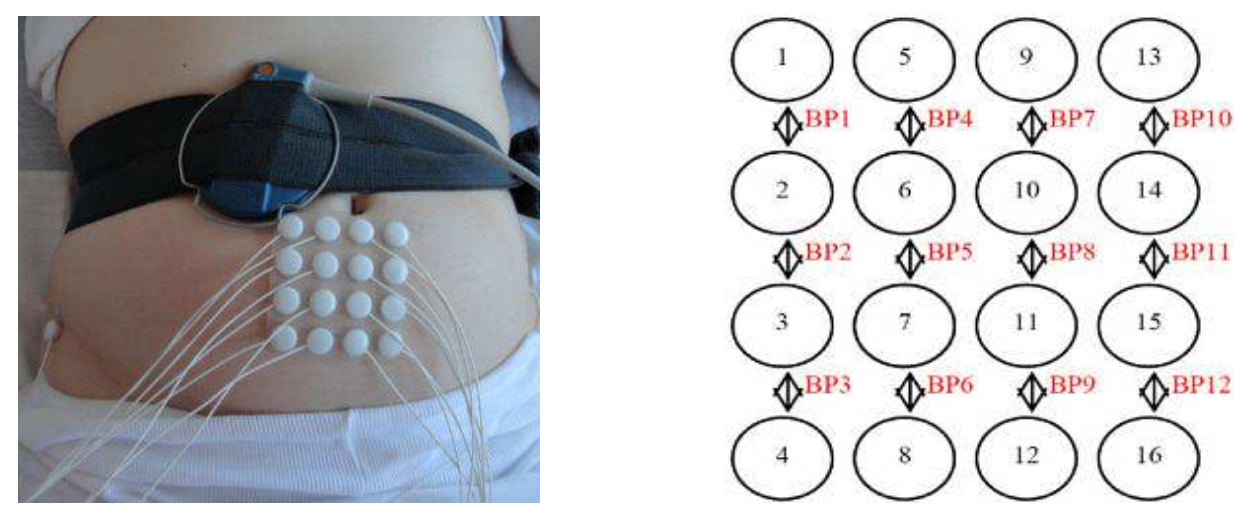

ए人9

Fig. 2. Electrode placement (left), monopolar configuration and the corresponding bipolar signals BPi (right). 


\section{Evolution}

Direct method, Without Noise

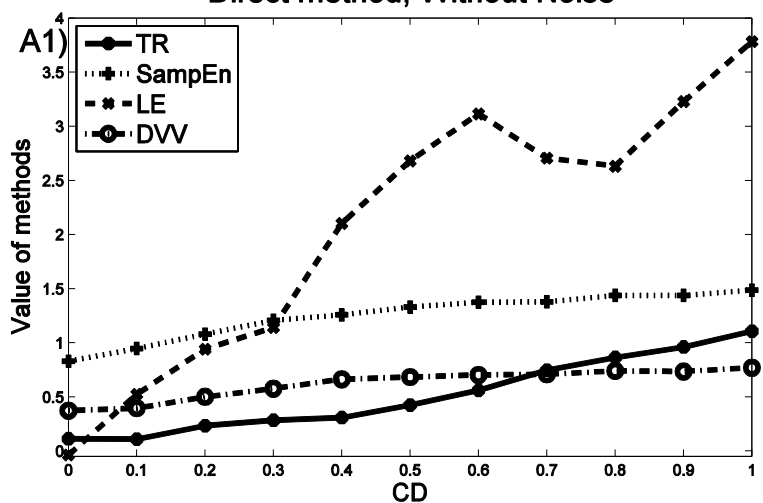

Direct method, With Noise

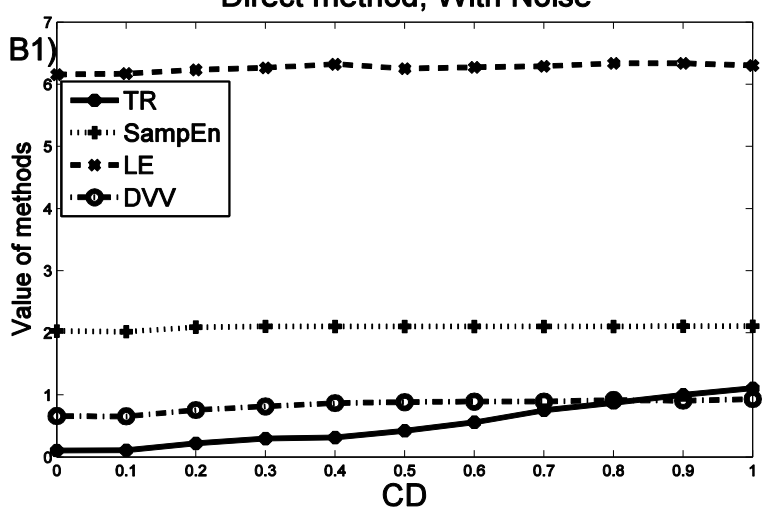

rql

rar

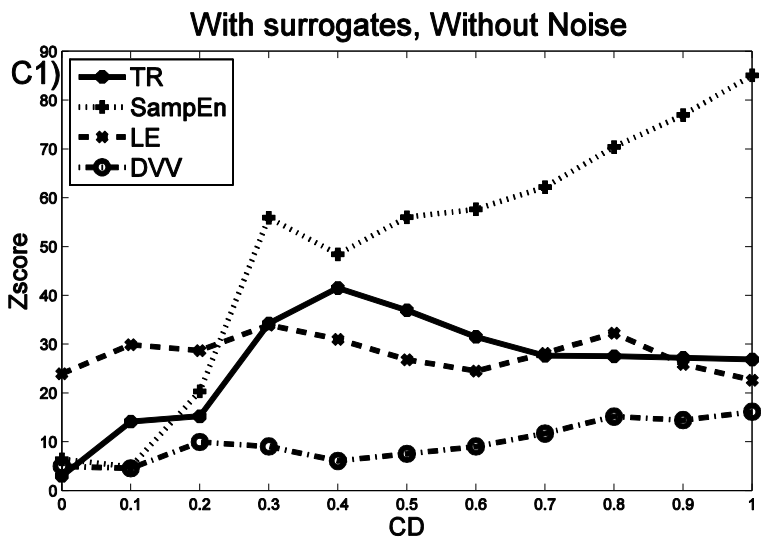

With surrogates, With Noise

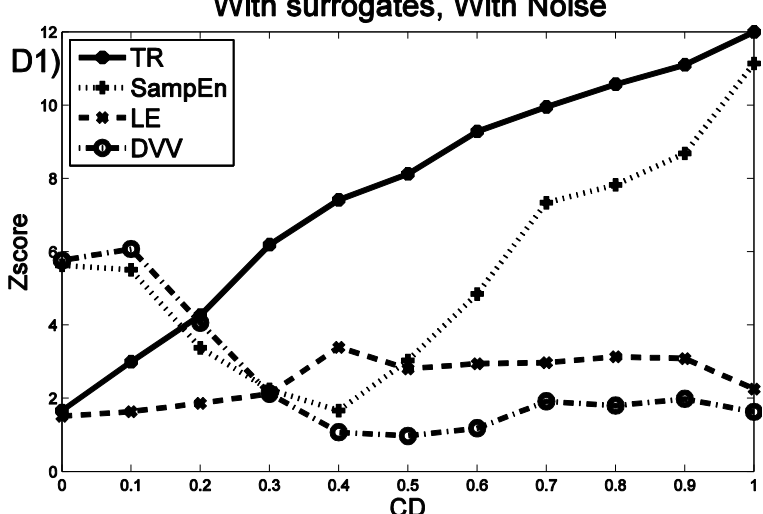

Error

Direct method, Without Noise

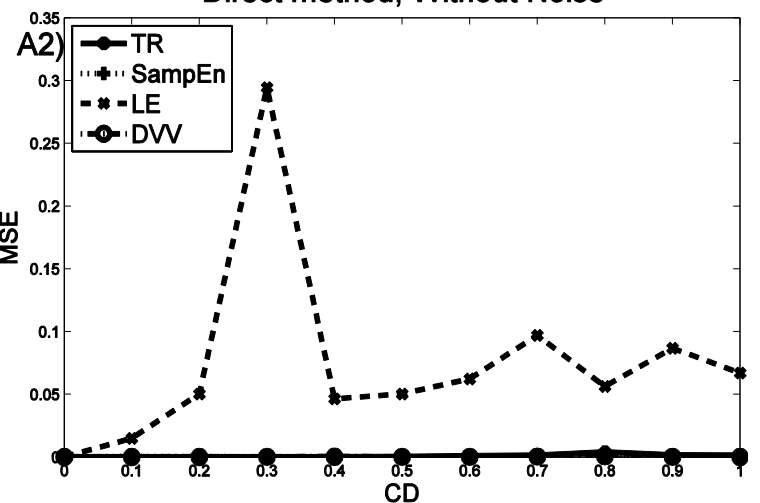

Direct method, With Noise

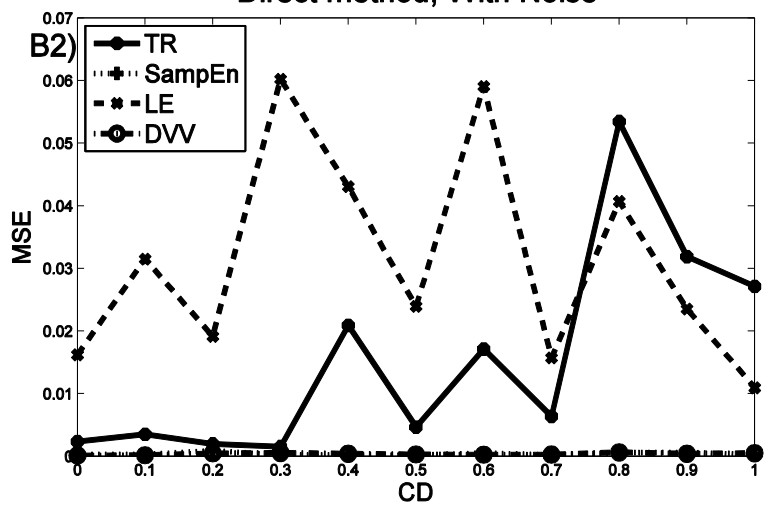

With surrogates, Without Noise

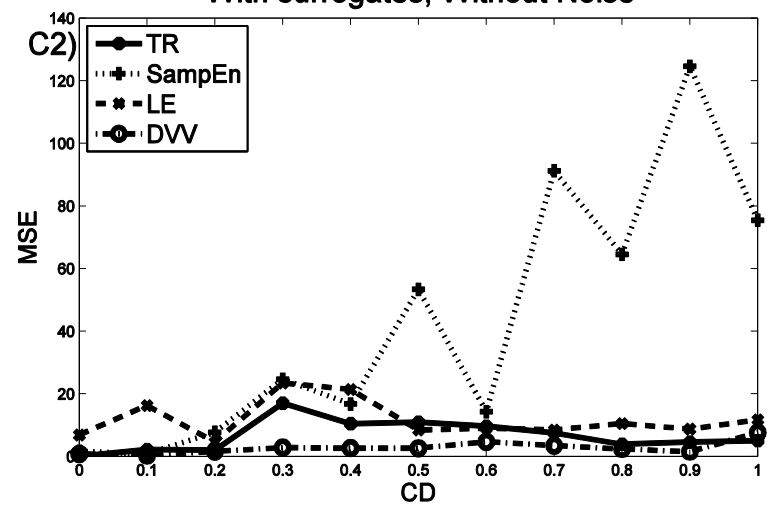

With surrogates, With Noise

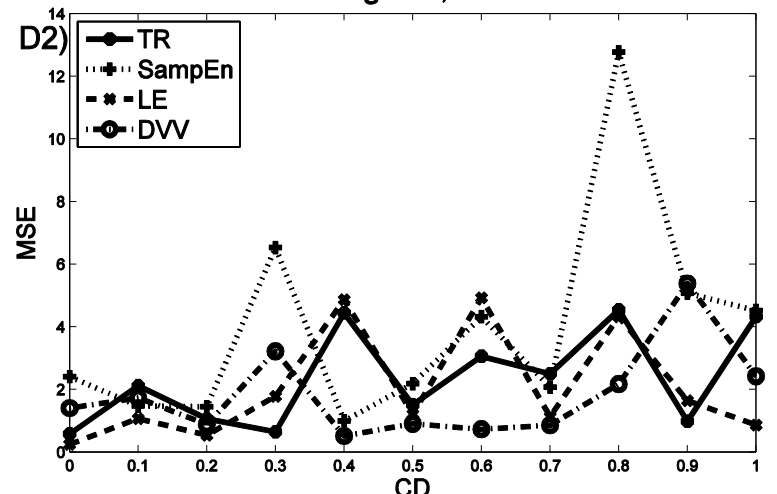

Fig. 3.Results obtained for Henon model using Monte-carlo simulation. On the left: Evolution of the methods with variable complexity in different cases. On the right: MSE of the methods function of complexity degree in different cases. (A) Direct method with no added noise, 

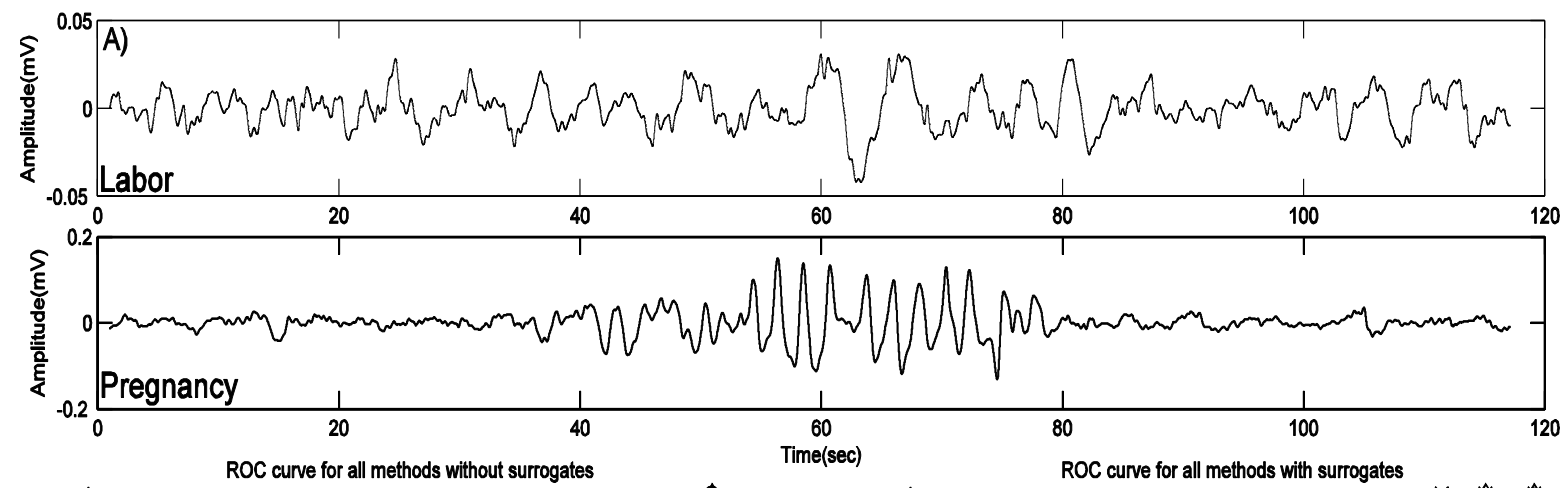

$\varepsilon \cdot r$
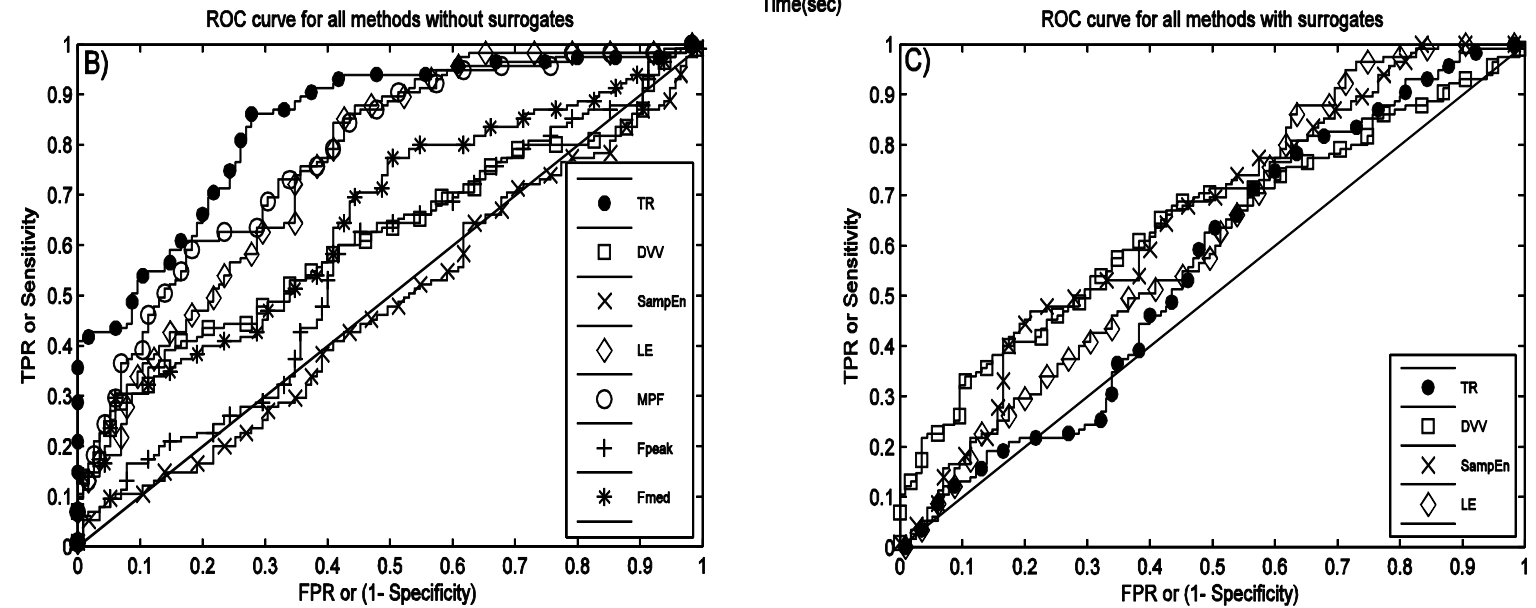

$\varepsilon$. $\quad$ Fig. 4. Example of ROC curves obtained for the detection of labor with the different linear and nonlinear methods. (A) Real Pregnancy and

乏.० Labor contractions, (B) Direct method, (C) With surrogate use. 
$\varepsilon \cdot 1$

TABLE I (Direct method)

$\varepsilon \cdot V$

Comparison of ROC curves for labor detection

$\varepsilon \cdot \wedge$

$\varepsilon \cdot 9$

Parameter

AUC

Specificity

Sensitivity

$\varepsilon 1$.

Time reversibility

0.842

0.721

0.860

Sample Entropy

0.478

0.382

0.643

\&1)

Lyapunov Exponent

$0.758 \quad 0.643$

0.756

हा

Delay Vector Variance

$0.615 \quad 0.582$

0.600

Mean Power Frequency

0.778

0.678

0.730

Peak Frequency

0.561

0.582

0.600

Median Frequency

0.654

0.556

0.704

₹10

E) 7

हाV

$\varepsilon 11$

E19

$\Sigma Y$.

EY

$\varepsilon r Y$

ETr

$\varepsilon Y \varepsilon$

EYo

EYT

$\varepsilon r V$

$\sum Y \wedge$

$\varepsilon Y q$ 
TABLE II (with surrogate use)

Comparison of ROC curves for labor detection

\begin{tabular}{lccc}
\hline \hline Parameter & AUC & Specificity & Sensitivity \\
\hline Time reversibility & 0.560 & 0.513 & 0.626 \\
Sample Entropy & $\mathbf{0 . 6 5 0}$ & $\mathbf{0 . 5 9 3}$ & $\mathbf{0 . 6 4 3}$ \\
Lyapunov Exponent & 0.614 & 0.591 & 0.530 \\
DVV & 0.642 & 0.573 & 0.669 \\
\hline
\end{tabular}

